\title{
EN BUSCA DE UNA SALUD MÁS PARTICIPATIVA: COMPARTIENDO DECISIONES DE SALUD
}

\author{
Paulina Bravo ${ }^{1,2, a}$, Aixa Contreras ${ }^{1, b}$, Lilisbeth Perestelo-Pérez ${ }^{3, c}$, Jeanette Pérez-Ramos ${ }^{4, d}$ \\ Germán Málaga ${ }^{5, e}$
}

\section{RESUMEN}

\begin{abstract}
El modelo de atención en salud paternalista está derivando hacia modelos más participativos, como lo es la toma de decisiones compartidas (TDC), en el que se considera al paciente como agente responsable y autónomo. La TDC representa un enfoque terapéutico en el que profesionales y pacientes comparten la mejor evidencia científica disponible para tomar una decisión, incorporando los valores y preferencias del paciente. Este intercambio de información puede facilitarse mediante el uso de herramientas de ayuda para la TDC, que han demostrado ser efectivas para mejorar el conocimiento, la satisfacción del paciente, y reducir el conflicto decisional. En este sentido, las habilidades comunicacionales ejercen un rol fundamental en el establecimiento de la relación profesional - paciente, facilitando el intercambio de información y preferencias de manera efectiva y respetuosa. Esta aproximación terapéutica podría apoyar la reducción de las disparidades en salud que prevalecen en Latinoamérica, al facilitar que las personas puedan participar informada y activamente en el cuidado de su salud.
\end{abstract}

Palabras clave: Toma de decisiones; Comunicación en salud; Medicina basada en evidencia (fuente: DeCS BIREME).

\section{LOOKING FOR A MORE PARTICIPATIVE HEALTHCARE: SHARING MEDICAL DECISION MAKING}

\begin{abstract}
The healthcare model is shifting from a paternalistic towards a more inclusive and participative approach, such as shared decision making (SDM). SDM considers patients as autonomous and responsible agents. SDM is a therapeutic approach where healthcare providers and patients share the best evidence available to make a decision according to the values and preferences of the patient. Decision aids are tools that can facilitate this information exchange. These tools help patients to increase knowledge about options, reduce decisional conflict and improve satisfaction. Additionally, communication skills play a key role within the professional-patient relationship, as they facilitate sharing information and preferences in an effective and respectful manner. This therapeutic approach could support the reduction of health inequalities that affect Latin America, as it promotes an active and informed participation of patients in their healthcare process
\end{abstract}

Key words: Decision making; Health communication; Evidence-based medicine (source: MeSH NLM).

\section{INTRODUCCIÓN}

En las últimas décadas se ha despertado el interés por involucrar a las personas en el cuidado de la salud. Así, los modelos de atención están cambiando, desde un enfoque más paternalista, donde el profesional de la salud es considerado el experto y quien acumula el mayor poder en la relación terapéutica hacia un enfoque más participativo que reconoce responsabilidades y obligaciones del paciente. Esto permite una relación más equilibrada, en términos de poder y autoridad, para el paciente y el profesional sanitario ${ }^{(1,2)}$.

En este contexto surge la toma de decisiones compartidas (TDC) como un modelo de atención que promueve el involucramiento del paciente en el cuidado de su salud ${ }^{(3,4)}$. Diversas iniciativas internacionales han demostrado que la participación de las personas en la

\footnotetext{
Escuela de Enfermería, Pontificia Universidad Católica de Chile, Chile.

School of Medicine, Cardiff University. Reino Unido.

Servicio de Evaluación del Servicio Canario de la Salud, Red de Investigación en Servicios de Salud en Enfermedades Crónicas (REDISSEC). España

Fundación Canaria de Investigación y Salud (FUNCIS). España.

Conocimiento y Evidencia (CONEVID), Universidad Peruana Cayetano Heredia. Lima, Perú.

Enfermera, doctora en Salud Pública; ${ }^{\text {b }}$ enfermera matrona, magister en Psicología Social Comunitaria; ${ }^{c}$ psicóloga, doctora en Psicología Clínica y de la Salud; ${ }^{\mathrm{d}}$ psicóloga; ${ }^{\mathrm{e}}$ médico internista, magister en Medicina

Recibido: 01-08-2013 Aprobado: 06-11-13
}

Citar como: Bravo P, Contreras A, Perestelo-Pérez L, Pérez-Ramos J, Málaga G. En busca de una salud más participativa: compartiendo decisiones de salud. Rev Peru Med Exp Salud Publica. 2013;30(4):691-7. 
toma de decisiones sobre su salud, y el apoyo brindado por los profesionales en este proceso, aumentan la satisfacción y pueden beneficiar algunos resultados de salud ${ }^{(5)}$.

Sin embargo, la mayoría de las experiencias en TDC provienen de Norteamérica y Europa. En el contexto de la VII Conferencia Internacional de TDC (7th ISDM conference), celebrada en Lima(Perú) en junio de 2013, los autores de este documento realizaron el primer seminario en español en TDC. Este artículo presenta los contenidos teóricos desarrollados durante el seminario que deben considerarse para la adopción e implementación de la TDC en la práctica clínica. Los temas incluyen una revisión de la medicina basada en evidencia, generalidades de la TDC, el uso de herramientas para el apoyo en la TDC y habilidades comunicacionales que ayudan a la práctica e implementación de la TDC.

\section{MEDICINA BASADA EN EVIDENCIA Y SU RELACIÓN DE MUTUA DEPENDENCIA CON LA TOMA DE DECISIONES COMPARTIDA}

Se acepta que la implementación y práctica de la MBE constituye la forma más racional y humana de proveer los cuidados de salud, siendo el principio de "utilización de la mejor evidencia disponible" (6) el más reconocido. No obstante, este ha sido mal utilizado o distorsionado, pues implica una aplicación y discusión limitada a los resultados de la investigación científica, disponible a una audiencia más académica, que la convierte en una medicina centrada en la investigación ${ }^{(7)}$. Adicionalmente, se ha reconocido que "la evidencia per se, no es suficiente para tomar una decisión", por lo que deben respetarse los valores y preferencias de las personas como parte del proceso de toma decisiones conjunta ${ }^{(6)}$.

En la práctica clínica, los profesionales de la salud requieren de la mejor evidencia disponible y de su experiencia para interpretar de manera adecuada los síntomas del paciente y las pruebas diagnósticas que permitan alcanzar un correcto diagnóstico. Pero, también se debe interpretar el dilema del paciente relacionado con su contexto cultural, social y económico, y solo así se podrá ofrecer la mejor alternativa terapéutica. Esto último hace un llamado para que los profesionales consideren los valores y preferencias del paciente, y la decisión se haga realmente dentro de los lineamientos filosóficos de la MBE ${ }^{(6)}$.

Los valores y preferencias implican las perspectivas, creencias, expectativas y metas para el cuidado de la salud y la vida en general; a la vez que deben tenerse en cuenta los beneficios, daños potenciales, costes e inconvenientes en la implementación de las decisiones ${ }^{\left({ }^{8}\right)}$.
La consideración de los valores y preferencias es aun más importante a la hora de implementar tratamientos para enfermedades crónicas, altamente dependientes de la adherencia al tratamiento; cuando el beneficio es escaso, como en intervenciones en las que el "número de pacientes a tratar" es muy alto para obtener un beneficio; o cuando la calidad de la evidencia es baja y los beneficios y riesgos deben ser sopesados cuidadosamente ${ }^{(9)}$. Contemplar los valores y preferencias sobre los tratamientos o pruebas diagnósticas supone un punto particularmente crítico, ya que estos pueden ser altamente variables entre los pacientes si tenemos en cuenta sus expectativas particulares, experiencias previas e idiosincrasia (10); o la probabilidad de que sus preferencias sean diferentes a las de los profesionales de la salud (11).

En el modelo convencional de práctica médica, también conocido como "paternalista", la dirección y cantidad de información fluye del médico o profesional de salud hacia el paciente, así como la deliberación sobre las opciones también la desarrolla el médico, quien finalmente tomará la decisión sin tener en cuenta los valores y preferencias de los pacientes (2). En este modelo, la decisión se fundamenta básicamente en recomendaciones de expertos, siguiendo evidencia de baja calidad o desactualizada en relación con la mejor evidencia científica disponible ${ }^{(12)}$. Es por ello que el modelo paternalista no es concordante con lo establecido por la MBE.

Por otro lado, el modelo de TDC promueve que "la cantidad y dirección de información, la información acerca de valores y preferencias, la deliberación y decisiones, ocurra con la participación de ambas partes; es decir, profesional de salud y paciente están completamente involucrados y actuando asociadamente" (13). Este modelo propugna como condición básica y elemental, que la decisión que se comparta deba ser de alta calidad y estrechamente relacionada con el uso de la mejor evidencia disponible. Para ello se han generado sistemas estándar de valoración de la evidencia (14) que permitan no solo que las recomendaciones tengan carácter de uniformidad, sino que el entendimiento de una evidencia de calidad y la fuerza de recomendación de una determinada intervención sean asequibles.

\section{GENERALIDADES EN LA TOMA DE DECISIONES COMPARTIDAS}

Las personas se enfrentan cada día a diversas decisiones, que van desde situaciones cotidianas como qué ropa usar cada mañana hasta las más complejas como tener hijos, cambiar de trabajo, etc. Las personas 
deciden entre una serie de opciones que incluyen un curso de acción o no acción frente a una circunstancia determinada ${ }^{(15)}$.

De forma similar, en el cuidado de la salud, las personas se enfrentan a distintas situaciones que requieren de la elección de una alternativa de tratamiento, diagnóstico o prevención, que se traduce en una decisión. Las circunstancias únicas de los usuarios de salud, el curso de una enfermedad y la falta de efectividad absoluta de las terapias y tratamientos disponibles hacen que las decisiones acerca de la salud y enfermedad de una persona se tornen difíciles. La incertidumbre a la que los profesionales de la salud se enfrentan en su práctica clínica hace necesario un curso de acción que incluya aquello que es importante para los pacientes, lo que ellos valoran y aprecian como importante ${ }^{(8,16)}$. Surge así la TDC como un modelo que promueve la participación de las personas en las decisiones relacionadas con su salud.

Una de las definiciones más aceptadas de la TDC es la presentada por Elwyn et al. en la que se considera que profesionales y pacientes comparten la mejor evidencia disponible al momento de tomar una decisión, los pacientes son apoyados para que consideren las opciones disponibles, y así poder tomar una decisión informada ${ }^{(17)}$.

La idea de involucrar a las personas en las decisiones de salud no es nueva. En el año 1982 se describió por primera vez el concepto shared decision making o toma de decisiones compartidas en un documento estadounidense acerca del consentimiento informado y aspectos éticos de la práctica clínica ${ }^{(18)}$. Este documento destacó por primera vez la importancia de involucrar a los pacientes y tomar decisiones compartidas a través de un proceso mutuo de respeto y compañerismo ${ }^{(18)}$, en el que ser reconozcan sus valores y preferencias.

Promover la participación de las personas en el cuidado de su salud se considera un imperativo ético. Así lo establece uno de los primeros llamados públicos para la implementación de la TDC, conocido como la Declaración de Salzburg (2010), donde los profesionales y pacientes son invitados a convertirse en coproductores de la salud (19). El componente ético de la TDC se basa en que los profesionales reconocen la autodeterminación del paciente como un objetivo a conseguir y facilitan el cumplimiento de ese objetivo ${ }^{(20)}$. Esto se consigue al respetar la autonomía de las personas, sin abandonarlas, sino acompañándolas en las decisiones relacionadas a su salud ${ }^{(19,20)}$.
Tabla 1. Elementos esenciales e ideales para la práctica de la toma de decisiones compartidas

\begin{tabular}{ll}
\hline \multicolumn{1}{c}{ Esenciales } & \multicolumn{1}{c}{ Ideales } \\
\hline Definir y explicar el problema & Definir roles \\
$\begin{array}{l}\text { Presentar las opciones } \\
\text { (pros y contras) }\end{array}$ & Presentar evidencia \\
$\begin{array}{l}\text { Explorar preferencias y valores } \\
\text { del paciente }\end{array}$ & $\begin{array}{l}\text { Acordar mutuamente la } \\
\text { decisión }\end{array}$ \\
$\begin{array}{l}\text { Explorar las capacidades y } \\
\text { habilidades del paciente }\end{array}$ & \\
Seguimiento & \\
\hline
\end{tabular}

Según Makoul y Clayman ${ }^{(18)}$, la práctica de la TDC tiene varios elementos esenciales y otros que idealmente debieran practicarse en la TDC. Estas características se presentan en la Tabla 1.

Si bien la TDC tiene un importante componente ético, diversas investigaciones han demostrado que el respetar la autonomía de las personas en relación a las decisiones en salud se traduce en una práctica clínica efectiva. Una revisión Cochrane demostró tras examinar 86 ensayos controlados aleatorizados que la TDC aumenta el conocimiento de los pacientes, ayudándolos a sentirse más seguros acerca de la decisión y, consecuentemente, más activos e involucrados en el cuidado de su salud ${ }^{(5)}$.

En Estados Unidos, en mayo de 2013 un grupo de informantes claves, investigadores y profesionales de la salud declararon en el "Aligning Incentives Statement" que a través de un esfuerzo colectivo es posible crear un sistema sanitario de cuidado compasivo y respetuoso, que invite y permita la participación activa de los usuarios y sus familias. Esto facilita el establecimiento de un compromiso entre el usuario y el profesional de salud, lo que se traduce en el aumento de los niveles de calidad, seguridad y eficiencia del cuidado, y así se logra la transformación del sistema de salud (21).

\section{IMPLEMENTACIÓN Y HERRAMIENTAS DE AYUDA PARA LA TOMA DE DECISIONES COMPARTIDAS}

Para favorecer el proceso de TDC se han desarrollado intervenciones conocidas como herramientas de ayuda para la toma de decisiones (HATD) que pueden ser útiles cuando existen varias opciones diagnósticas o terapéuticas disponibles para una determinada condición médica, aportando información objetiva sobre las diferentes opciones y los resultados de salud potencialmente esperados (riesgos y beneficios), en un lenguaje y formato adecuado para el paciente ${ }^{(17)}$. 
Las HATD se basan en la mejor evidencia científica disponible y se utilizan para complementar el asesoramiento clínico, ayudar a pacientes y familiares a sentirse más informados y capaces de tomar decisiones de acuerdo a sus valores y preferencias. Pueden utilizarse durante o después de la consulta y su uso puede variar según su extensión y formato en el que estén disponibles: tarjeta de decisión, folleto, cuadernillo, vídeo, DVD, página Web o programa interactivo ${ }^{(5)}$.

El número y calidad de las HATD disponibles está aumentando en diferentes sistemas sanitarios y su uso comienza a generalizarse, especialmente en instituciones de Norteamérica y Europa, mientras que en Latinoamérica comienza a ser un área de interés ${ }^{(22,23)}$. Con el fin de definir criterios estándar para el desarrollo y evaluación de las HATD, la organización profesional International Patient Decision Aid Standards (IPDAS) ha establecido criterios que permiten valorar la calidad de las HATD en relación con su contenido, proceso de desarrollo y evaluación (4).

Las HATD incrementan el nivel de conocimiento de los pacientes sobre la enfermedad y los tratamientos disponibles, proporcionando una mejor percepción de los riesgos, lo que disminuye el conflicto decisional y aumenta el nivel de participación de los pacientes ${ }^{(5)}$. Los pacientes informados tienden a elegir procedimientos menos invasivos, inician los tratamientos de forma más precoz y con mayor adherencia, mostrando un mayor nivel de satisfacción con el proceso y menos ansiedad en general ${ }^{(5,24)}$. Otros factores que confieren utilidad a las HATD están relacionados con su capacidad para favorecer la mejora en el uso apropiado de los recursos y la reducción de costes innecesarios ${ }^{(25)}$.

Por tanto, la participación activa de los pacientes en las decisiones relacionadas con su salud y el uso de HATD podría favorecer una mejoría en la atención sanitaria. No obstante, es importante tener en cuenta las posibles barreras y facilitadores relacionadas con el paciente, los profesionales y el propio contexto a la hora de diseñar, evaluar e implementar estas herramientas en la práctica clínica. La falta de tiempo y recursos, el escaso deseo de participación, la baja capacidad de comprensión de algunos pacientes, o la falta de familiaridad de los profesionales con el modelo de TDC podrían entorpecer su uso e implementación (26).

Para subsanar algunas de estas limitaciones, se destaca la importancia de capacitar y formar a los profesionales en un estilo de atención asistencial centrado en el paciente, con el fin de establecer estilos de comunicación que favorezcan el modelo de TDC ${ }^{(27-29)}$.

\section{HABILIDADES COMUNICACIONALES PARA LA TOMA DE DECISIONES COMPARTIDAS}

La TDC es en esencia un espacio personal de reflexión y análisis que supone un apoyo de otras personas significativas, donde los profesionales sanitarios pueden brindar al paciente no solo la mejor información científica disponible para enfrentar su situación de salud o enfermedad, sino que deben ser capaces de generar un espacio interpersonal de respeto, escucha y acogida que haga sentir al paciente comprendido y aceptado.

La comunicación cumple un importante rol en el espacio terapéutico de la TDC. El uso correcto de las habilidades comunicacionales es fundamental para la presentación de información y opciones en la TDC, así como en la exploración de los valores y preferencias del paciente. Tal es la relevancia de la comunicación para la TDC, que uno de los innovadores modelos para la implementación de TDC en la práctica clínica sugiere que esta puede realizarse a través de tres pasos "conversacionales": conversación de oportunidades; conversación de opciones, y conversación de decisión (choice talk, option talk, decision talk) ${ }^{(20)}$.

La comunicación es considerada un proceso de interacción que pone toda su atención en la relación que ocurre entre los comunicantes, ya que ambos son influidos y afectados por la interacción comunicativa. Esto realza que cualquier acto, más allá de las palabras, posee un poderoso poder comunicativo, y esforzarse por entenderlos como un todo, se constituye en el mayor de los desafíos ${ }^{(30)}$. Es frecuente encontrar que a la base de numerosos conflictos interpersonales una de las personas ha sido capaz, o tan solo ha querido atender exclusivamente a las palabras, dejando de lado el poder comunicativo que poseen las claves no verbales que completan ese mensaje (31). El tono de voz, la mirada o los gestos que acompañan una frase, puede cambiar completamente la dirección del contenido que se ha entregado a través de las palabras ${ }^{(32)}$. Esto tiene un efecto directo en la TDC, pues el paciente podría percibir la información entregada por el profesional como sesgada o incompleta, desvirtuando el proceso colaborativo y respetuoso de la decisión. Esto sugiere que los profesionales de salud requieren un entrenamiento permanente para mejorar la capacidad de comprender lo que el otro vive, y darse cuenta de la influencia que la propia conducta posee en la interacción con el paciente.

Uno de los elementos básicos es la escucha activa, que permite transformar al profesional en un apoyo real, para que el paciente aumente su autocomprensión, explore su incertidumbre, potencie sus propios recursos de 
Tabla 2. Elementos que favorecen la escucha activa para la práctica de toma de decisiones compartidas

\begin{tabular}{ll}
\hline \multicolumn{1}{c}{ Elemento facilitador } & \multicolumn{1}{c}{ Argumento } \\
\hline $\begin{array}{l}\text { Evitar la selectividad en la } \\
\text { escucha }\end{array}$ & $\begin{array}{l}\text { La tendencia a escuchar solo lo que se quiere escuchar, ya sea para confirmar un prejuicio o } \\
\text { por creer que ya se conoce el tema que se está comunicando, impide atender el verdadero re- } \\
\text { lato emocional que acompaña los hechos y que dan forma real a lo que se está comunicando }\end{array}$ \\
$\begin{array}{l}\text { Tomar el tiempo necesario } \\
\text { para entender lo que se está } \\
\text { escuchando }\end{array}$ & $\begin{array}{l}\text { La necesidad de responder algo apropiado o bloquear algún sentimiento o tema que está } \\
\text { emergiendo, lleva muchas veces a interrumpir el relato y a reflejar poco interés }\end{array}$ \\
$\begin{array}{l}\text { Proporcionar señales concretas } \\
\text { de escucha y comprensión }\end{array}$ & $\begin{array}{l}\text { La retroalimentación oportuna del contenido y de los sentimientos que se han comunicado } \\
\text { demuestra atención. Podemos apoyarnos en las señales como movimientos de cabeza que } \\
\text { afirmen el relato, o palabras como "entiendo..., "continúe por favor..., "sígame contando...", } \\
\text { entre otras. Generar contacto visual con el paciente, le brinda seguridad y aceptación. Una } \\
\text { postura corporal relajada y simétrica con el interlocutor refleja horizontalidad en la relación }\end{array}$ \\
\hline $\begin{array}{l}\text { Observar la conducta no verbal } \\
\text { Movimientos, vestimenta, gestos, tono, ritmo y congruencia del relato, expresiones emocio- } \\
\text { nales y reacciones ante determinados temas }\end{array}$ \\
\hline
\end{tabular}

ayuda, y defina con libertad la mejor opción disponible, ante el problema de salud que enfrenta. Escuchar activamente, debe considerar un espacio de apertura a los sentidos en que no solo se recoge el contenido verbal entregado sino se atiende al contenido emocional y gestual que lo acompaña. Escuchar activamente es una de las formas más eficaces de respeto, pues ser escuchado se traduce en una sensación de ser valorado y comprendido en plenitud, generando las mejores condiciones para otorgar una ayuda eficaz ${ }^{(33)}$.

Pese a que cualquier análisis de variables que afectan el proceso de escucha va a ser limitado, la Tabla 2 señala algunas claves que favorecen el proceso de escucha activa.

Toda acción comunicativa en TDC debe orientarse a ofrecer al usuario un espacio donde pueda expresar libremente aprehensiones, sentimientos o conflictos que pueda tener ante una decisión. Por lo tanto, la base será establecer con naturalidad una relación empática, no moralizante y respetuosa.

\section{CONCLUSIONES}

La TDC surge como una alternativa efectiva para mejorar el sistema sanitario que tradicionalmente se ha basado en un modelo paternalista. A través de un cuidado respetuoso, que considera a la persona (o paciente) como un miembro activo y responsable de las decisiones en salud, los profesionales sanitarios pueden comunicar riesgos y alternativas terapéuticas, considerando aquello que es importante para el paciente de acuerdo a sus valores y preferencias.

La evidencia científica tiene un rol fundamental para informar de la mejor manera al paciente que se enfrenta a una decisión, y las HATD surgen como elementos facilitadores para la presentación de opciones en un lenguaje familiar y acorde a las necesidades del paciente. Esto podría tener un efecto en la actual lucha por erradicar las desigualdades en salud que afectan a la región. Así, por ejemplo, aquellas personas con bajo nivel educacional que se enfrenten a una decisión relacionada con su salud, pueden acceder a la mejor evidencia, de manera que sea entendible para ellos, mientras sus valores personales son destacados y considerados por el profesional de la salud. Todo esto es posible a través de una comunicación efectiva basada en la empatía y la escucha activa que permite al profesional entender la situación del paciente, mientras este último se siente aceptado, respetado y apoyado.

Si bien la implementación de modelos de TDC aún es tarea pendiente en países latinoamericanos (22,23,34), y su implementación en modelos sobrecargados de pacientes, sin ayudas tecnológicas o con pacientes con bajo nivel educativo en salud, podría parecer una quimera, su implementación en otras latitudes ha demostrado no incrementar sustancialmente el tiempo del encuentro clínico, en cambio, mejora la interacción entre el paciente y el médico interlocutor, mejora el conocimiento de la enfermedad, mejora la adherencia, y reduce la ansiedad generada, lo que resulta de invalorable ayuda, en especial en el control de enfermedades crónicas ${ }^{(5,8)}$, estas son situaciones que deben ser exploradas y desarrolladas.

Resulta evidente que aún existen una serie de limitantes y barreras, como las mencionadas, relacionadas a las características de nuestro sistema de salud, tanto publico como privado, pero además, habría que tener en consideración, la aún baja difusión y practica de TDC en nuestro medio, con la consiguiente necesidad del desarrollo de HATD, herramientas para su aplicación y la 
ausencia de desarrollo de habilidades comunicacionales requeridas de manera especial.

La conferencia realizada en Perú ha sido una oportunidad para observar la realidad global y latinoamericana en relación al modelo de TDC. Futuras investigaciones deberían explorar las barreras y facilitadores identificadas por los pacientes, legisladores, investigadores y profesionales de la salud, que podrían afectar la adopción de un modelo de atención centrado en la persona y en su activa participación en las decisiones de salud, la búsqueda de las estrategias más apropiadas de implementación, además de identificar el efecto que esto podría tener en el alcance de una salud más justa y equitativa para la región.

Contribuciones de autoría: PB, AC, LPP, JPR y GM participaron en la concepción, diseño del artículo, redacción y revisión crítica del artículo, todos los autores aprobaron la versión final para publicación.

Fuentes de financiamiento: autofinanciado.

Conflictos de interés: los autores declaran no tener conflictos de interés.

\section{REFERENCIAS BIBLIOGRÁFICAS}

1. Wanless D. Securing good health for the whole population. Final Report. London: Department of Health; 2004.

2. Emanuel EJ, Emanuel LL. Four models of the physician-patient relationship. JAMA. 1992;267(16):2221-6.

3. Coulter A. Paternalism or partnership? Patients have grown up-and there's no going back. BMJ. 1999;319(7212):71920.

4. Elwyn G, O'Connor A, Stacey D, Volk R, Edwards A, Coulter A, et al. Developing a quality criteria framework for patient decision aids: online international Delphi consensus process. BMJ. 2006;333(7565):417.

5. Stacey D, Bennett CL, Barry MJ, Col NF, Eden KB, Holmes-Rovner $\mathrm{M}$, et al. Decision aids for people facing health treatment or screening decisions. Cochrane Database Syst Rev. 2011;5(10): CD001431. doi: 10.1002/14651858.CD001431.pub3.

6. Guyatt G, Haynes B, Jaeschke, Meade MO, Wilson M, Montori V, et al. The philosophy of Evidence Based Medicine. User's Guides to the Medical Literature, A manual for Evidence Based Clinical Practice. 2nd ed. New York: Mc Graw Hill; 2008.

7. Montori V, Guyatt G. Progress in evidence-based medicine. JAMA. 2008;300(15):1814-6. doi: 10.1001/ jama.300.15.1814.

8. Charles C, Gafni A, Whelan T. Decision-making in the physicianpatient encounter: revisiting the shared treatment decision-making model. Soc Sci Med. 1999;49(5):651-61.
9. Montori V, Gafni A, Charles C. A shared treatment decision-making approach between patients with chronic conditions and their clinicians: the case of diabetes. Health Expect. 2006;9(1):25-36.

10. MacLean S, Mulla S, Akl EA, Jankowski M, Vandvik PO, Ebrahim S, et al. Patient values and preferences in decision making for antithrombotic therapy: a systematic review: Antithrombotic Therapy and Prevention of Thrombosis, 9th ed: American College of Chest Physicians Evidence-Based Clinical Practice Guidelines. Chest. 2012;141(2 Suppl):e1S-23S. doi: 10.1378/ chest.11-2290.

11. Devereaux PJ, Anderson DR, Gardner MJ, Putnam W, Flowerdew GJ, Brownell BF, et al. Differences between perspectives of physicians and patients on anticoagulation in patients with atrial fibrillation: observational study. BMJ. 2001;323(7323):1218-22.

12. Antman E, LauJ, KupelnickB, Mosteller F, Chalmers T. A comparison of results of meta-analyses of randomized control trials and recommendations of clinical experts. Treatments for myocardial infarction. JAMA. 1992;268(2):240-8.

13. Charles C, Whelan T, Gafni A. What do we mean by partnership in making decisions about treatment? BMJ. 1999;319(7212):780-2.

14. Guyatt G, Oxman AD, Akl EA, Kunz $\mathrm{R}$, Vist G, Brozek J, et al. GRADE guidelines: 1. Introduction-GRADE evidence profiles and summary of findings tables.J Clin Epidemiol. 2011;64(4):38394. doi: 10.1016/j.jclinepi.2010.04.026.
15. Joseph-Williams N, Edwards A, Elwyn G. The importance and complexity of regret in the measurement of 'good' decisions: a systematic review and a content analysis of existing assessment instruments. Health Expect. 2011;14(1):59-83. doi: 10.1111/j.1369-7625.2010.00621.x.

16. Elwyn G, Frosch D, Rollnick S. Dual equipoise shared decision making: definitions for decision and behaviour support interventions. Implement Sci. 2009;4:75. doi: 10.1186/1748-59084-75.

17. Elwyn G, Frosch D, Volandes AE, Edwards A, Montori VM. Investing in deliberation: a definition and classification of decision support interventions for people facing difficult health decisions. Med Decis Making. 2010;30(6):701-11. doi: $10.1177 / 0272989 X 10386231$.

18. Makoul G, Clayman ML. An integrative model of shared decision making in medical encounters. Patient Educ Couns. 2006;60(3):301-12.

19. Salzburg Global Seminar. Salzburg statement on shared decision making. BMJ. 2011;342:d1745. doi: 10.1136/ bmj.d 1745 .

20. Elwyn G, Frosch D, Thomson R, JosephWilliams N, Lloyd A, Kinnersley P, et al. Shared decision making: a model for clinical practice. J Gen Intern Med. 2012;27(10):1361-7.

21. Informed Medical Decisions Foundation. The Aligning Incentives Statement [Internet]. Boston, MA: Informed Medical Decisions Foundation; c2011-2013 [citado el 
11 de julio de 2013]. Disponible en: http://informedmedicaldecisions. org/past-sdm-events/aligningincentives-for-patient-engagementenabling-widespread-implementationof-shared-decision-making/ the-aligning-incentives-statement/.

22. Abreu MM, Battisti R, Martins RS, Baumgratz TD, Cuziol M. Shared decision making in Brazil: history and current discussion. Z Evid Fortbild Qual Gesundhwes. 2011;105(4):2404. doi: 10.1016/j.zefq.2011.04.009.

23. Bravo P, Cabieses B, Bustamante C, Campos S, Stacey D. Shared decision making in Chile: supportive policies and research initiatives. Z Evid Fortbild Qual Gesundhwes. 2011;105(4):2548. doi: 10.1016/j.zefq.2011.04.007.

24. Knops AM, Legemate DA, Goossens A, Bossuyt PM, Ubbink DT. Decision aids for patients facing a surgical treatment decision: a systematic review and meta-analysis. Ann Surg. 2013;257(5):860-6. doi: 10.1097/ SLA.0b013e3182864fd6.

25. Veroff D, Marr A, Wennberg DE. Enhanced support for shared decision making reduced costs of care for patients with preference-sensitive conditions. Health Aff. 2013;32(2):285-93. doi: 10.1377/hlthaff.2011.0941.
26. Legare F, Ratte S, Gravel K, Graham ID. Barriers and facilitators to implementing shared decisionmaking in clinical practice: update of a systematic review of health professionals' perceptions. Patient Educ Couns. 2008;73(3):526-35. doi: 10.1016/j.pec.2008.07.018.

27. Legare F, Ratte S, Stacey D, Kryworuchko J, Gravel K, Graham ID, et al. Interventions for improving the adoption of shared decision making by healthcare professionals. Cochrane Database Syst Rev. 2010;12(5):CD006732. doi: 10.1002/14651858.CD006732.pub2..

28. Legare F, Turcotte S, Stacey D, Ratte S, Kryworuchko J, Graham ID. Patients' perceptions of sharing in decisions: a systematic review of interventions to enhance shared decision making in routine clinical practice. Patient. 2012;5(1):1-19. doi: 10.2165/11592180-00000000000000.

29. May CR, Mair F, Finch T, MacFarlane A, Dowrick C, Treweek $S$, et al. Development of a theory of implementation and integration: Normalization Process Theory. Implement Sci. 2009;4:29. doi: 10.1186/1748-5908-4-29..
30. López A, Parada A, Simonetti F. Psicología de la comunicación. 5a ed. Santiago: Universidad Católica de Chile; 2009.

31. Otero E. Las cuentas pendientes de la teoría de la comunicación. Punto Cero. 2010;15(21):7-15.

32. Simonetti F. Juegos de comunicación. 3a ed. Santiago: Ediciones Universidad Católica de Chile; 2007.

33. Brusco A. Humanización de la asistencia al enfermo. Santander: Sal Térrea; 2000.

34. Perestelo-Perez L, Rivero-Santana A, Perez-Ramos J, Gonzalez-Lorenzo M, Roman JG, Serrano-Aguilar P. Shared decision making in Spain: current state and future perspectives. Z Evid Fortbild Qual Gesundhwes. 2011;105(4):28995. doi: 10.1016/j.zefq.2011.04.013.

Correspondencia: Germán Málaga, Correo electrónico:german.malaga@upch.pe Dirección: Calle Chavin 159. CC Monterrico. Santiago de Surco, Lima, Perú Teléfono: 51-992768300

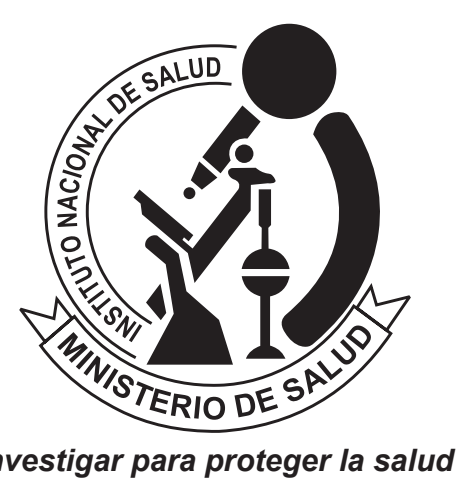

REVISTA PERUANA DE MEDICINA EXPERIMENTAL Y SALUD PÚBLICA CUMPLIENDO SUS METAS Y PROYECTÁNDOSE AL FUTURO

\section{Visite los contenidos de la revista en: www.ins.gob.pe/rpmesp}

